\title{
AVOIDING CANNIBALISATION AND/OR COMPETITOR REACTION IN PLANAR SINGLE FACILITY LOCATION
}

\author{
Frank Plastria* \\ Vrije Universiteit Brussel
}

(Received May 8, 2004)

\begin{abstract}
A single facility has to be located in the plane in competition with fixed existing facilities, some belonging to the same chain, the others to competing chains. Demand points patronise the facility to which they are most attracted. Attraction is increasing with the quality of the facility and decreasing with its distance to demand.

We study the lexicographic optimisation problem with primary objective to maximise the total market share of the chain, and secondarily to minimise the market losses of friendly facilities and/or the risk that competing facilities would raise their current quality in order to gain back lost customers.
\end{abstract}

Keywords: Facility planning, planar location, competition, cannibalisation, maxcovering, mincovering

\section{Introduction}

In competitive location theory a basic question is locating a new facility in a market where other facilities are already present, see e.g. the surveys $[5,9,22]$ for models of many different kinds. These existing facilities either belong to the same chain or to some competing chains, and all can have differing characteristics, like size, product mix, price, service level, ..., summarised as their quality (or attractiveness), which determines, together with the distance to the facility, the attraction customers feel towards the facility.

The primary aim of starting up a new facility is to ensure the largest possible total market share for its entire chain. In our model a binary ([12]) or all-or-nothing rule is assumed, in which each customer fully patronises the facility to which it is attracted most. It is well known (see e.g. [4]) that in the planar demand point setting we consider, there usually exists a whole region of optimal locations.

This raises the question, posed in [15], which of these optimal locations to prefer. It was argued there that a location central to the set of optimal locations would be preferable to a boundary one, and an algorithm was described to construct a most central point. No real rationale was given for this centrality idea, so no clear objective was described for this secondary optimisation step.

In this paper we introduce several proposals for such objectives which are of direct

*The research for this paper was partially done in the framework of the research project BEC2002-01026 of the Ministry of Science and Technology of Spain, in part financed by the European Regional Development Fund (ERDF). It was written while visiting Nanzan University, Nagoya, Japan, for the International Workshop on Urban Operations Research, December 2003. We gratefully acknowledge the organisers for their generous support. 
practical interest and may be combined in sequential manner. We also describe algorithmic approaches to determine a (usually) unique solution optimising the resulting lexicographic goal.

The first secondary objective is aimed at counteracting the loss in market share of friendly facilities (of the same chain), due to the introduction of the new facility. This effect, known in retailing as cannibalisation (see [11]), if present, may result in strong opposition from the managers of the friendly facilities. It is therefore quite interesting to see that the possibility exists to minimise this effect while still keeping the maximal possible total market share of the chain. It turns out that this secondary objective usually still yields an infinity of optimal locations.

We therefore introduce yet another objective, taking into consideration the fact that the new facility also causes losses in market share for some competing facilities. Most probably these facility-managers will try to win their lost customers back. In practice it is usually a lot easier to modify the facility characteristics than to move it to another place, or to open new facilities, which would lead to Stackelberg type location models as studied and described in $[6,8,12]$. Here we rule out the latter, and assume that the facility will rather consider improving its quality, so as to attract more customers, possibly even ones they were not patronised by before. But of course this should remain economically feasible for them. Therefore we propose on the one hand to look at a worst case future in which all competing facilities would raise their quality to the highest level possible to them and to secure the maximal market share the new facility would then still be able to obtain. On the other hand we introduce a final touch-up step aimed at discouraging the competition to attempt recovery of lost customers, by making this as costly as possible to the competing chains.

\section{Market Share Maximisation}

Let $A \subset \mathbb{R}^{2}$ denote the finite set of customer(groups). Each customer $a \in A$ has a known location $x_{a} \in \mathbb{R}^{2}$ and a strictly positive weight $\omega_{a}$, indicating its demand or buying power, e.g. the population or total wealth represented by customer(group) $a$.

The new facility will have to share the market with a finite set of facilities already active in the market. The set $E$ of these existing facilities is subdivided into friendly facilities $(F)$, belonging to the same chain, and competing facilities $(C)$ of other chains. Existing facility $e \in E$ is located at site $x_{e}$ and has a known quality $\alpha_{e} \in \mathbb{R}$.

All distances will be measured by a fixed norm with an easy description of the unit ball. In this paper we will mainly focus on euclidean distance, but the ideas are readily extended to rectangular or polyhedral distance.

The tastes of a customer $a \in A$ are expressed by a function $\mathcal{A}_{a}(\alpha, d)$ giving the attraction it feels to a facility of quality $\alpha$ at distance $d$. The possibly $+\infty$-valued function $\mathcal{A}_{a}$ : $\mathbb{R} \times \mathbb{R}^{+} \longrightarrow \mathbb{R} \cup\{+\infty\}$ is assumed to be nondecreasing and continuous in $\alpha$ for each fixed $d \geq 0$, and nonincreasing and upper-semicontinuous in $d$ for each fixed $\alpha$. See [24] for more details and examples of attraction functions. Notice that several customers with different tastes may be present at a same location.

One important case we will use here to focus on as motivating example, is a millpricing situation (see [14]), where each facility fixes a unit price $p$ for their product and customers take into account travel cost to the facility to obtain it. Quality of the facility, as seen by customer $a$, is then opposite to this price, i.e. $\alpha=-p$, and attraction is accordingly given as the opposite of the full price, inclusive transport. With a standard linear transportcost assumption, with unitcost $t_{a}$, we obtain attraction functions of type 
$\mathcal{A}_{a}(\alpha, d)=-\left(p+t_{a} d\right)=\alpha-t_{a} d$

Customers behave deterministically by patronising the facility they are most attracted to. This model allows us to describe the current allocation of the market as follows. For each customer $a \in A$, we may determine the existing facility $e(a)$ they patronise in the current market situation by

$$
e(a) \stackrel{\text { def }}{=} \operatorname{argmax}\left\{\mathcal{A}_{a}\left(\alpha_{e}, d\left(x_{a}, x_{e}\right)\right) \mid e \in E\right\}
$$

For a new facility with quality $\alpha$ located at point $x$ we may then calculate the critical distance $r_{a}$ within which the new facility should be located in order to win over (capture) customer $a$ from the facility it currently patronises, by

$$
\begin{aligned}
r_{a} & \stackrel{\text { def }}{=} \min \left\{d \in \mathbb{R}^{+} \mid \mathcal{A}_{a}(\alpha, d) \geq \mathcal{A}_{a}\left(\alpha_{e(a)}, d\left(x_{a}, x_{e(a)}\right)\right)\right\} \\
& =\min \left\{d \in \mathbb{R}^{+} \mid \forall e \in E: \mathcal{A}_{a}(\alpha, d) \geq \mathcal{A}_{a}\left(\alpha_{e}, d\left(x_{a}, x_{e}\right)\right)\right\}
\end{aligned}
$$

which is well defined by the assumptions on $\mathcal{A}_{a}$.

The location $x$ must be chosen within a feasible region $R \subset \mathbb{R}^{2}$. The description of $R$ should allow certain queries to be answered efficiently; these are

- it must be possible to check easily whether a point belongs to $R$

- one can determine the points of $R$ 's boundary at a given distance of a given point, or at equal distance of two given points, and the number of such intersection points is uniformly bounded.

For example any finite union of planar regions bounded by line segments or circular arcs is acceptable.

To simplify some notations we will write $n=|A|$. The complexity of $R$ will be denoted by $r$ in the following sense: the number of intersection points mentioned above is assumed of $O(r)$.

The primary objective is to maximise the chain's market share. An increase in the chain's market share will only be due to those customers switching over from some competing facility to the chain, because of the advent of the new facility. Customers already patronising an existing friendly facility may or may not switch over to the new facility, but this will not matter for the chain's market share, so should not be taken into account here: they will be considered in the next section.

As explained in [24], in order to guarantee existence of optimal solutions, we also (have to) assume novelty orientation in chain switching: any customer having to choose between a competing facility and the new facility, because equally attracted to both, will opt for the new facility.

Therefore maximisation of the new market share gained by the new facility reduces to a constrained maximal covering problem in the plane with feasible region $R$, and as demand points the set

$$
A^{C} \stackrel{\text { def }}{=} e^{-1}(C)=\{a \in A \mid e(a) \in C\}
$$

of potential new customers for the chain (all customers currently patronising a competing facility), each weighted by $w_{a}$, and captured when within radius $r_{a}$.

This problem can be quite efficiently solved in $O(n(n+r) \log (n+r))$ using the following slight modification of the algorithm of [6] to take the feasible region $R$ into account.

Consider the example shown in figure $1 . A^{C}$ consists of 11 customers, 5 with weight 1 (small black points), and 6 with weight 2 (large black points), while the feasible region $R$ 


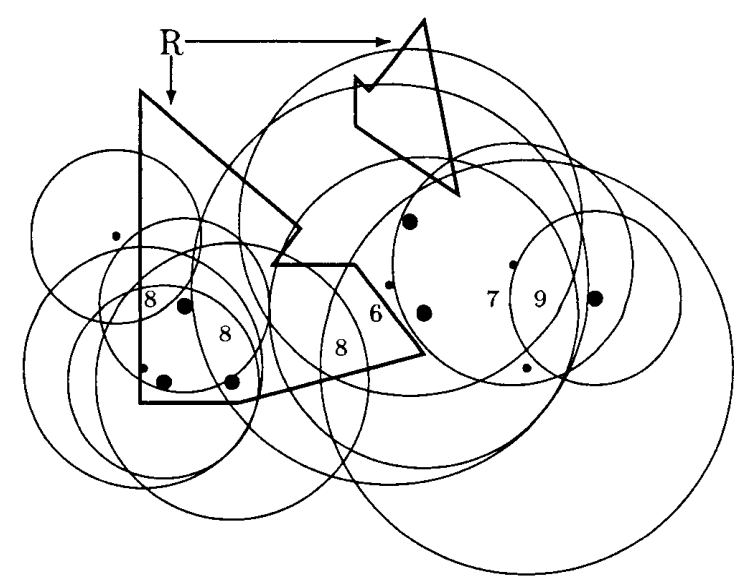

Figure 1: A maxcovering problem with constraints

consists of two (nonconvex) polygons. For each customer its capturing disk with center $x_{a}$ and radius $r_{a}$ is drawn. Notice that by novelty orientation the capturing disks are closed. These disks split the plane into separate cells within which the covered weight is constant, equal to the sum of weights of all customers inside whose capturing disk the cell lies. We can see that the maximal value of 9 is reached in one, totally infeasible cell. The next value in rank is 8 , reached in three of the cells. Since they all meet the feasible region, the optimal solution set is the union of the part of these three cells within $R$.

Evidently the optimal value is always reached in some (probably several) intersection point of the circles, and/or in an intersection point of a circle and $R$ 's boundary. We can construct all these points by simple intersection between two circles or between one circle and the boundary of $R$. On each separate circle we may calculate all its $O\left(\left|A^{C}\right|+r\right)$ intersection points with other circles and with $R$ 's boundary in $O\left(\left|A^{C}\right|+r\right)$ time; a circular sort of this list in $O\left(\left(\left|A^{C}\right|+r\right) \log \left(\left|A^{C}\right|+r\right)\right)$ then allows to calculate all covered weights reached at each of these intersection points in $O\left(\left|A^{C}\right|+r\right)$, by starting at one of the intersection points, for which the covered weight is determined directly in $O\left(\left|A^{C}\right|\right.$, and from there sequentially moving along the list and adding (subtracting) the weight of the circle that is entered (left). Each time a point on $R$ 's boundary is crossed we change the feasibility status, storing all those intersection points with status feasible and highest recorded covered weight. Repeating this $O\left(\left(\left|A^{C}\right|+r\right) \log \left(\left|A^{C}\right|+r\right)\right)$ procedure for the $O\left(\left|A^{C}\right|\right)$ circles we will have in store all feasible intersection points which give the optimal feasible coverage. Note in passing that in fact a full census of all intersection points and their corresponding covered weight is obtained; this will be useful in section 5 .

The total region of optimal solutions may then be reconstructed in a final step as follows. For each optimal feasible intersection point in store (there are $O\left(\left|A^{C}\right|\left(\left|A^{C}\right|+r\right)\right)$ such), find the intersection of the capturing disks of all customers covered by this point, intersected with $R$. The result will be a new region $R_{1}$, possibly consisting of several connected components, each bounded by circle-arcs and/or parts of $R$ 's boundary. Notice that each component will be reconstructed as many times as it has intersection points on its boundary, and that these intersection points are exactly the breakpoints in $R_{1}$ 's boundary. So the description of $R_{1}$ is of $O\left(\left|A^{C}\right|\left(\left|A^{C}\right|+r\right)\right)=O(n(n+r))$. The total complexity of the algorithm is $O\left(\left|A^{C}\right|\left(\left|A^{C}\right|+r\right) \log \left(\left|A^{C}\right|+r\right)\right)=O(n(n+r) \log (n+r))$. 


\section{Cannibalisation Reduction}

Let us now move to a second objective to be achieved within the region $R_{1}$ of primary optimal solutions. We want to minimise the cannibalisation. In other words, we want to minimise the total weight of customers who patronised a friendly chain, but will switch over to the new facility.

Therefore we face a minimal covering problem constrained to $R_{1}$, with demand points the set

$$
A^{F} \stackrel{\text { def }}{=} e^{-1}(F)=\{a \in A \mid e(a) \in F\}
$$

each with weight $w_{a}$ and covered as soon as the new facility lies within distance $r_{a}$.

This new problem can be solved in a totally analogous way as in the previous section, suitably adapted to minimisation instead of maximisation. Notice that to guarantee existence of optimal solutions, we now should assume within chain conservatism: any customer having to choose between a friendly existing facility and the new facility, because equally attracted to both, will not switch. In other words, all capturing disks are open disks.

We construct all these capturing disks centered at points $x_{a}\left(a \in A^{F}\right)$ and radius $r_{a}$. This gives again a diagram with cells within which the covered weight will be constant. We seek those cells which intersect $R_{1}$ and yield lowest covered weight.

Because $R_{1}$ is of complexity $r_{1}=O(n(n+r))$, this can be found in $O\left(n\left(n+r_{1}\right) \log (n+\right.$ $\left.\left.r_{1}\right)\right)=O\left(n^{2}(n+r) \log (n+r)\right)$ time by the same algorithm as described above, but storing now the feasible intersection points with lowest found covered weight.

The procedure will result in a set $R_{2}$, as union of cells intersected with $R_{1}$, so having again a boundary consisting of linear and circular arc segments, of total description complexity $r_{2}=O\left(n\left(n+r_{1}\right)\right)=O\left(n^{2}(n+r)\right)$ (although in practice it will be much smaller).

\section{Competitor Reaction Risk Avoidance}

Usually $R_{2}$ will again be infinite, although smaller, so there may still be room for optimisation of an additional objective.

Let us then look at the effect of the new facility's entry as seen by the competing facilities. Some of them will have lost customers, and might try to win back market share. To this end they have two non-exclusive options: relocate and/or improve their quality. However it usually is prohibitively expensive to relocate an existing facility, and therefore investment into improving quality is much more likely to be attempted, as long as the involved costs remain reasonable.

We propose to investigate the chain's policy of reducing as much as possible the risk connected with this competitor's reaction, by making it as hard as possible for competition.

We will again consider this as a two step process. First we want to secure as much as possible of the captured demand which cannot be taken back because it would be uneconomical to the competition. This corresponds to a maxmin worst case approach to the uncertainty of reaction. Second, for the demand which could be lost back to a competitor, we finally indicate a way to make this as hard as possible to them.

\subsection{Securing approach in worst case scenario}

Our first worst case type objective leads to a series of max covering type problems as follows.

For every competing facility $c \in C$ an upper bound on reachable quality $\alpha_{c}^{\text {max }}$ (possibly $+\infty$ ) is assumed to be known, determined extraneously by the local conditions at that facility's site, as what is reasonably possible.

We now consider each cell $\sigma$ of $R_{2}$ in turn. 
First determine the set $A_{\sigma}^{c a p} \subset A^{C}$ of customers captured from the competition by the new facility if it were located in $\sigma$, which is given by

$$
A_{\sigma}^{c a p} \stackrel{\text { def }}{=}\left\{a \in A^{C} \mid \forall x \in \sigma \quad \forall c \in C: \mathcal{A}_{a}\left(\alpha, d\left(x_{a}, x\right)\right) \geq \mathcal{A}_{a}\left(\alpha_{c}, d\left(x_{a}, x_{c}\right)\right)\right\}
$$

(note that this set is constant over $\sigma$ ). For each customer $a \in A_{\sigma}^{\text {cap }}$ determine the radius $r_{a}^{\prime}$ within which the new facility must lie from $a$ in order to still capture it even if all competing facilities would upgrade to their maximal possible quality, i.e.

$$
r_{a}^{\prime} \stackrel{\text { def }}{=} \min \left\{d \in \mathbb{R}^{+} \mid \forall c \in C: \mathcal{A}_{a}(\alpha, d) \geq \mathcal{A}_{a}\left(\alpha_{c}^{\max }, d\left(x_{a}, x_{c}\right)\right)\right\}
$$

Consider then the maxcovering problem constrained to $\sigma$ with demand points the set $A_{\sigma}^{c a p}$, weights $w_{a}$ and covering radius $r_{a}^{\prime}$.

We finally retain as optimal the best covering value so obtained over all cells $\sigma$ of $R_{2}$, and reconstruct the optimal solution set $R_{3}$ as the union of optimal solution sets within $\sigma$, for each $\sigma$ where this covering value was reached. As explained before this can be done in $O\left(n\left(n+r_{2}\right) \log \left(n+r_{2}\right)\right)=O\left(n^{3}(n+r) \log (n+r)\right)$ time.

It may be observed that since the cells $\sigma$ tend to be quite small, this step might be totally ineffective. Indeed, if every competing facility $c \in C$ currently operates with a quality $\alpha_{c}$ sufficiently far below their maximum possible quality $\alpha_{c}^{\max }$, then each cell $\sigma$ will tend to lie fully outside distance $r_{a}^{\prime}$ for all $a \in A_{\sigma}^{c a p}$. In this case the maxcovering problem suggested here will be totally degenerate, having constant value 0 over the whole feasible region $R_{2}$, and therefore $R_{3}=R_{2}$. It is only when some of the competing facilities already offer close to their uppermost quality that we may observe some reduction of the optimal region in this step.

Also, it may often not be possible to estimate close upper bounds $\alpha_{c}^{\text {max }}$ by lack of adequate information on the competition's efficiency. This would mean that one will have to work on the basis of wide over-estimations for these values, again leading to large $r_{a}^{\prime}$ values, rendering this step quite useless.

\subsection{Risk avoidance}

Therefore we now turn to attempting to minimise the risk of competitor's upgrading of their quality. To this end we consider the decision problem each competitor has whether to upgrade its quality or not.

In principle this should be answered by a cost-benefit analysis, weighing off the costs involved in upgrading and the corresponding benefits. The customer attraction model used here is able to measure the benefit part because we assumed the attraction functions $\mathcal{A}_{a}$ of all customers to be known, as well as the quality $\alpha_{c}$ of each competing facility $c \in C$. Indeed this allows us to measure the effects obtained by any competing facility when modifying its quality. We cannot, however, reasonably expect to have adequate information about the costs involved in upgrading this quality, since this kind of data is directly related to competing chain's accounting, which normally should be considered as confidential.

Therefore we prefer to turn the question around as follows. Instead of considering a competing facility increasing its quality, let us consider lowering the quality $\alpha$ of the new facility. At any fixed location $x \in R_{3}$ of the new facility we may determine what is the minimum quality $\alpha^{\text {min }}(x)$ necessary to keep the covered weight unchanged. If $\alpha^{\text {min }}(x)$ is strictly lower than the current $\alpha$, any competitor will have to raise its current quality in order to regain some of its customers lost to the new facility, and the larger the difference $\alpha-\alpha^{m i n}(x)$, the larger this rise in quality needed, and the harder it will be for the competition 
to do so. It thus seems an appropriate strategy for the new facility to look for a location which maximises this difference.

Hence we propose the following optimisation problem, as a proxy to the cost-benefit approach:

or equivalently

$$
\max _{x \in R_{3}}\left(\alpha-\alpha^{\min }(x)\right)
$$

$$
\min _{x \in R_{3}} \alpha^{\min }(x)
$$

A precise definition of $\alpha^{\text {min }}(x)$ must now be obtained. Note first that the remaining feasible region $R_{3}$ consists of a union of cells $\sigma^{\prime}$, which are all a subset of some cell $\sigma$ of $R_{2}$. For each such cell $\sigma^{\prime}$ of $R_{3}$ we determine the set $A_{\sigma^{\prime}}^{\text {recap }} \subset A^{C}$ of customers captured from some competitor by the new facility if it were located in $\sigma^{\prime}$, but which may possibly be recaptured by this competitor after a sufficient raise of its quality. If $\sigma$ is the cell of $R_{2}$ containing $\sigma^{\prime}$, this is the subset of $A_{\sigma}^{c a p}$ which excludes all the customers secured in previous step. It is therefore given by

$$
A_{\sigma^{\prime}}^{r e c a p} \stackrel{\text { def }}{=}\left\{a \in A_{\sigma}^{c a p} \mid \forall x \in \sigma^{\prime} \quad d\left(x_{a}, x\right)>r_{a}^{\prime}\right\}
$$

Note that when previous securing step was not taken, things become slighter simpler since we may work at this point within region $R_{1}$ and with $A_{\sigma}^{\text {recap }}=A_{\sigma}^{c a p}$.

For each such customer $a \in A_{\sigma^{\prime}}^{r e a p}$ we also determine the decisive attraction $\mu_{a}$ needed to win this customer over, i.e.

$$
\mu_{a} \stackrel{\text { def }}{=} \mathcal{A}_{a}\left(\alpha_{e(a)}, d\left(x_{a}, x_{e(a)}\right)\right)
$$

For any $x \in \sigma^{\prime}$ we may then obtain $\alpha^{m i n}(x)$ by

$$
\alpha^{\min }(x) \stackrel{\text { def }}{=} \min \left\{\alpha \mid \forall a \in A_{\sigma^{\prime}}^{\text {recap }}: \mathcal{A}_{a}\left(\alpha, d\left(x_{a}, x\right)\right) \geq \mu_{a}\right\}
$$

Introduce now the decisive quality function $\mathcal{B}_{a}(d)$, defined in [24] by the property

$$
\mathcal{B}_{a}(d) \leq \alpha \Longleftrightarrow \mathcal{A}_{a}(\alpha, d) \geq \mu_{a}
$$

$\mathcal{B}_{a}(d)$ indicates, as a function of $d$, the quality threshold above which customer $a$ at distance $d$ is captured. This value is uniquely defined, but possibly $+\infty$, because of the nondecrease and upper semicontinuity assumed of $\mathcal{A}_{a}(\alpha, d)$ for fixed $d$. It was shown in [24] that under mild conditions the decisive quality function $\mathcal{B}_{a}$ is nondecreasing and lower-semicontinuous in $d$. For example, using the mill-pricing attraction function $\mathcal{A}_{a}(\alpha, d)=\alpha-t_{a} d$ discussed in section 2 , and solving the equality $\mathcal{A}_{a}(\alpha, d)=\mu_{a}$ for $\alpha$, we obtain the decisive quality function $\mathcal{B}_{a}(d)=\mu_{a}+t_{a} d$.

As in [24], we rather need its composite with the distance to customer $a$, i.e. $\mathcal{D}_{a}(x)=$ $\mathcal{B}_{a}\left(d\left(x_{a}, x\right)\right.$ ), which was shown to be a quasiconvex and lower-semicontinuous function of $x$, and even convex (and hence continuous) as soon as $\mathcal{A}_{a}$ is quasiconcave (for more details on quasiconvexity and quasiconcavity see [1]). We may now write

$$
\alpha^{\text {min }}(x)=\min \left\{\alpha \mid \forall a \in A_{\sigma^{\prime}}^{\text {recap }}: \alpha \geq \mathcal{D}_{a}(x)\right\}=\max \left\{\mathcal{D}_{a}(x) \mid a \in A_{\sigma^{\prime}}^{\text {recap }}\right\}
$$

and our final risk proxy minimisation problem becomes

$$
\min _{\sigma^{\prime} \subset R_{3}} \min _{x \in \sigma^{\prime}} \max _{a \in A_{\sigma^{\prime}}^{\text {recap }}} \mathcal{D}_{a}(x)
$$


On each cell $\sigma^{\prime} \subset R_{3}$ we must therefore solve a quasiconvex minimax problem. When $\sigma^{\prime}$ is convex we might use some general quasiconvex minimisation algorithm like [19] or the more recent method described in [17], and when nonconvex some global optimisation method like $[13,20]$. Such methods are, however, probably much too demanding.

In $[2,3]$ it was shown that when the feasible region $\sigma^{\prime} \subset \mathbb{R}^{2}$ is convex, any optimal solution to such a problem is also the optimal solution to a similar minmax problem defined by a subset of $A_{\sigma^{\prime}}^{\text {recap }}$ of cardinality at most 3 . We conjecture that even for the possibly nonconvex $\sigma^{\prime}$ we have here (compare the proofs of similar results in [23]), that all locally optimal solutions are found among following points

- any solution in the interior of $\sigma^{\prime}$ of the equations

$$
\mathcal{D}_{a_{1}}(x)=\mathcal{D}_{a_{2}}(x)=\mathcal{D}_{a_{3}}(x)
$$

for some triplet of points $\left\{a_{1}, a_{2}, a_{3}\right\} \subset A_{\sigma^{\prime}}^{\text {recap }}$

- a solution on the boundary of $\sigma^{\prime}$ of the equations

$$
\mathcal{D}_{a_{1}}(x)=\mathcal{D}_{a_{2}}(x)
$$

for some pair of points $\left\{a_{1}, a_{2}\right\} \subset A_{\sigma^{\prime}}^{\text {recap }}$

- any breakpoint on the boundary of $\sigma^{\prime}$

Anyway, this set should always include some very serious candidates for the optimal solution, and enumerating it should form an excellent heuristic, if not an exact optimisation method.

It may be noted that obtaining the solutions of the two first types may be done using standard mathematical software. In the particular case of the mill-pricing attraction mechanism with equal transport costs, the first subproblem is directly related to Apollonius tenth problem, asking for constructing the center of circumcircles of three given circles, a classical problem from planar geometry, recently studied from the computational point of view in $[16]$.

\section{Concluding Remarks and Extensions}

We have presented several different secondary objectives supplementing the initial maxcovering objective, which may be used in a sequential way as described here, or separately, and shown that corresponding optimisation is algorithmically feasible.

However, for problems with many demand points, as is usually the case in practice, the regions of optimal solutions may quickly become very small, and there will not remain enough room to obtain significant improvements on the secondary objectives.

More important effects will be obtainable when one does not insist upon absolute optimality, and allow for some amount of loss in the primary objective. The methodology clearly still applies when the feasible region $R_{1}$ of optimal solutions to the primary maxcovering goal is replaced in the next step by the level set at some slightly lower level than the maximum. This level set will also consist of a union of cells like $R_{1}$.

One might even do this repeatedly to obtain a trade-off curve between level in primary objective and degree of cannibalisation. It is to be noted that the captured weight may only take a finite number of different capture values, and these are the only levels to be considered. A sorting of these values will enable a sequential and exhaustive treatment of decreasing levels of capture, which may be stopped at any minimal level requested, or needed to remain economically feasible. The obtained trade-off curve then also allows to select an optimal profit solution, the profit not only including the income due to the captured weight, 
but also possible costs due to the amount of cannibalisation, e.g. compensation to be paid to cannibalised friendly facilities.

Similarly the risk of competitor reaction may be lowered this way at the expense of some lesser income. All these variants are left for future work.

It should be observed that cannibalisation reduction is not always an issue. Cannibalisation is of course always inexistent when the chain is a newcomer in the market, and hence no friendly facilities are present. But also in non-franchising cases where all facilities of a chain are operated by fixed wage personnel, cannibalisation is normally unimportant. In all such cases the objective in section 3 is irrelevant, and we may simply take $R_{2}=R_{1}$.

The competitor's reaction avoidance strategies seem more widely applicable. Here we have only considered the static response case, in which the competitors are allowed to adapt their quality once after entry of the new facility. Instead of such a one-round type of game, one might also study the outcome of a resulting quality game between new and competing facilities and the equilibrium to which it might tend. Such models are currently under study following up on the research initiated in [10].

\section{References}

[1] M.Avriel, W.E.Diewert, S.Schaible and I.Zhang: Generalized concavity (Plenum, New York,1988)

[2] E.Carrizosa and F.Plastria: On minquantile and maxcovering optimisation. Mathematical Programming, 71 (1995), 101-112.

[3] E.Carrizosa and F.Plastria: Polynomial algorithms for parametric minquantile and maxcovering planar location problems with locational constraints. TOP, 6 (1998), 179-194.

[4] T.Drezner: Locating a single new facility among existing unequally attractive facilities. Journal of Regional Science, 34 (1994), 237-252.

[5] T.Drezner: Competitive facility location in the plane. in Z. Drezner (ed.): Facility Location. A survey of applications and methods (Springer, Berlin, 1995), 285-300.

[6] Z.Drezner: On a modified one-center problem. Management Science, 27 (1981), 848851.

[7] Z.Drezner: Competitive location strategies for two facilities. Regional Science and Urban Economics, 12 (1982), 485-493.

[8] H.A.Eiselt and G.Laporte: Sequential location problems. European Journal of Operational Research, 96 (1996), 217-231.

[9] H.A.Eiselt, G.Laporte and J.-F.Thisse: Competitive location models: a framework and bibliography. Transportation Science, 27 (1993), 44-54.

[10] M.D.Garcia Pérez, P.Fernandez Hernandez and B.Pelegrín: On price competition in location-price models with spatially separated markets. TOP, 12 (2004), 351-374.

[11] A.Ghosh and C.S.Craig: FRANSYS: a franchise distribution system location model. Journal of Retailing, 67 (1991), 466-495.

[12] S.L.Hakimi: Locations with spatial interactions: competitive locations and games. In R.L.Francis and P.B.Mirchandani (eds.): Discrete Location Theory (Wiley, New-York, 1990), 439-478.

[13] P.Hansen, D.Peeters, D.Richard and J.-F.Thisse: The minisum and minimax location problems revisited. Operations Research, 33 (1985), 1251-1265. 
[14] P.Hansen, D.Peeters and J.-F.Thisse: The profit-maximizing Weber problem. Location Science, 3 (1995),67-85.

[15] J.Karkazis: Location of a facility in a competitive environment: a planar-based approach. Studies in Locational Analysis, 7 (1994), 77-90.

[16] D.-S.Kim, D.Kim, K.Sugihara and J.Ryu: Apollonius tenth problem as a point location problem. In V.N.Alexandrov, J.J.Dongarra, B.A.Juliano, R.S.Renner and C.J.K.Tan (eds.), Computational Science - ICCS 2001, (Lecture Notes in Computer Science, Springer, Heidelberg, 2001), 728-737.

[17] K.C.Kiwiel: Convergence and efficiency of subgradient methods for quasiconvex minimization. Mathematical Programming, 90 (2001), 1-25.

[18] A.Mehrez and A.Stulman: The maximal covering location problem with facility placement on the entire plane. Journal of Regional Science, 22 (1982), 361-365.

[19] F.Plastria: The minimisation of lower subdifferentiable functions under nonlinear constraints, an all feasible cutting plane method. Journal of Optimisation Theory and Applications, 57 (1988), 463-484.

[20] F.Plastria: GBSSS, the generalized big square small square method for planar single facility location. European Journal of Operational Research, 62 (1992), 163-174.

[21] F.Plastria: Profit maximising single competitive facility location in the plane. Studies in Locational Analysis, 11 (1997), 115-126.

[22] F.Plastria: Static competitive facility location: an overview of optimisation approaches. European Journal of Operational Research, 129 (2001), 461-470.

[23] F.Plastria and E.Carrizosa: Undesirable facility location with minimal covering objectives. European Journal of Operational Research, 119 (1999), 158-180.

[24] F.Plastria and E.Carrizosa: Optimal location and design of a competitive facility. Mathematical Programming, 100 (2004), 247-265.

Frank Plastria

MOSI - Department of Mathematics, Operational Research and Information Systems for Management Vrije Universiteit Brussel

Pleinlaan 2

B1050 Brussels, Belgium

E-mail: Frank.Plastria@vub.ac.be 